\title{
Bio-energetics
}

No particularly sophisticated techniques had been used to elucidate the essential elements of the two complementary mechanisms of photosynthesis and respiration which are engaged in the production of the energy carrier ATP and which constitute the basis of life. Nevertheless these techniques offer new means of revealing details of some of the still unknown stages in these processes. The pulsed laser had already made possible the use of precise flash illumination of biological specimens, and had helped to improve the signal/noise ratio in the requisite spectographic analyses.

The processes involved in the pro- duction of ATP by respiration are the reverse of those in photosynthesis. The active membrane in which the energy of sunlight is converted into chemical energy - the thylakoid is about $40 \AA$ thick and contains several hundred pigment cells (chlorophyll). The energy transfer from the solar input is accomplished by vectorial transport across this membrane of electrons from $\mathrm{H}_{2} \mathrm{O}$ molecules. A potential gradient develops and induces a flow of protons across the membrane into the enzyme responsible for the production of the energyrich ATP. There is therefore also a $\mathrm{pH}$ gradient across the membrane. The mechanism for splitting the $\mathrm{H}_{2} \mathrm{O}$ molecule and other fine details are still unknown but measurements have confirmed the basic findings of vectorial electron transfer, proton into enzyme migration and the need for the co-operation of two chlorophyll centres. It has been possible to induce part of such processes in membrane enclosed vesicles by the external application of a voltage and exposure to sunlight. From thermodynamic considerations the efficiency of such a photosynthesis process could reach $70 \%$, but in nature, the overall efficiency amounts to a mere fraction of this. Energy production is only an incidental part in the grand scheme of life and the survival of the species.

\section{Simon Newman}

\section{Heavy lons}

In modern physics, theories and models often make predictions that cannot be tested because they require conditions that are far out of experimental reach. This is especially true for nuclear physics. "Classical" nuclear physics usually is dealing with nuclear matter near its ground state: the excitation energies are normally quite low, the angular momenta are small and the densities are about the equilibrium density. For these conditions there exist quite successful models that are able to describe the gross structures (e.g. the liquid drop model), but there are also very elaborate models predicting details like the nuclear deformation and transition probabilities (e.g. the interacting boson (1B) model).

These more sophisticated models have been tested against the available data, where absolute transition matrix elements are usually known for not more than three to five nuclear levels. The deformation is usually only known for the ground-state in odd nuclei and for the first $2^{+}$state in even nuclei. Thus the comparison between experiment and nuclear models has had a relatively weak basis, especially as the significance of each data point is questionable because of the great experimental difficulties. Intrinsic and experimental difficulties limit the accuracy to such an extent that the deformation of excited states can be determinated to only about
$10 \%$, whereas for a transition matrix element $\pm 1 \%$ is an excellent result.

With the newly established powerful particle accelerators, exciting new classes of experiments can be performed and it was natural that topics covered mainly either experiments performed at these machines or new theoretical approaches to the experimental data yielded there. To broaden the basis of experimental data, it is promising to investigate very high energetic nuclear collisions or collisions between very heavy nuclei. The first type of experiment can be performed at Berkeley or Dubna. The machine at Dubna e.g. accelerates light nuclei such as ${ }^{16} \mathrm{O}$ up to an energy of $5 \mathrm{MeV} /$ nucleon. In reactions between such high energetic projectiles and target nuclei, densities of several times the normal density are reached. For these conditions such spectacular things like phase transitions and pion condensation are expected to occur. Even the explosion of the projectile inside the target nucleus has been observed. Understanding the physics under such extreme conditions seems to be only at the beginning. Nevertheless a lot of phenomena can be explained e.g. by a hydrodynamical model, that fits the experimental data surprisingly well, considering the simplicity of the modelling.

For the second class of experiments a new powerful tool is given by the UNILAC heavy ion accelerator in Darmstadt. This machine accelerates any known stable ion to energies well above the Coulomb barrier even when uranium is the target, (5 to $6 \mathrm{MeV} /$ nucleon). It should be emphasised also that this facility is not only used for nuclear physics research. The scale of experiments stretches from atomic physics to elementary particle physics, from quantum electrodynamics to the search for super-heavy elements. Using the UNILAC in nuclear physics it is now feasible for the first time to study the lead-uranium system slightly below the Coulomb barrier-performing multiple Coulomb excitation (MCE) experiments - or even above the barrier.

The great advantage of lead as a projectile lies not only in the heightened excitation probability, that is dependent on the charge number of the projectile but especially also on the rise of intrinsic angular momentum that can be transferred. In a classical picture, the angular momentum is proportional to the nuclear deformation of the target nucleus and the charge of the projectile. Quantum calculations yield essentially the same result but give only 80 to $90 \%$ of the classical value. For $\mathrm{Pb}$ shot on $\mathrm{U}$, a maximum value of $34 \hbar$ is expected in an MCE experiment. Unfortunately not only the desired effects are increased but a strong Doppler broadening due to the high recoil energy goes against the use of heavy ions as projectiles. The broadening is governed by the angular distribution of the target nuclei recoiling into vacuo, 
the angles being completely determined by kinematics.

In an experiment reported by D. Schwalm, this simple correlation was used to get rid of the Doppler broadening in a particle- $\gamma$ coincidence experiment. The scattered projectiles were detected in a position sensitive detector, covering a big solid angle. From the scattering angle, the Doppler shift energy was calculated using zero energy loss kinematics which is an excellent approximation because of the high bombarding energies. The Doppler shift itself was calculated relativistically. With that information a corrected spectrum was constructed. The energy resolution is about $3 \mathrm{keV}$ instead of the $50 \mathrm{keV}$ obtained in a direct measurement and this figure can still be improved.

With this refined experimental technique MCE experiments were performed in the actinide region to determine the reduced electrical quadrupole transitions $B(E 2, I \rightarrow I+2)(I=$ even) between successive states. The analysis is straightforward because the excitation mechanism, pure Coulomb interaction, is completely understood. In addition, for a heavy ion experiment, the well known Winther de Boer computer code that calculates the excitation cross sections semi classically, can be used. Thus the $B(E 2)$ values between two successive states can be determined from the intensity of the corresponding line in the spectrum. The number of available $B(E 2)$ values is now pushed to ten or more, which poses a severe test for nuclear models. Although the IB model is quite successful at the lowest levels, there are indications that it fails to explain the trend in the $B(E 2)$ values going up the yrast band. It must be admitted however, that the number of levels excited in experiments so far must be increased by one or two, to have a conclusive result. These improvements are expected in the near future.

\section{Yrast Traps}

A second group of experiments exploiting nuclear fusion processes are used in the search for the so called yrast traps, isomeric states to which the highly excited nucleus decays. The investigated nuclei with mass $A \cong 150$ were produced in a fusion process between a target nucleus of mass $A \cong 110$ and a projectile of mass $A \cong 50$. For a long time yrast traps have been predicted in this mass region. The nucleus formed, leaves the target foil with a high recoil velocity at approx. $0^{\circ}$ and after some $10 \mathrm{~cm}$ of flight is caught by another foil, which is surrounded by a big Nal detector that covers a solid angle of almost $4 \pi$. Through a hole in the $\mathrm{Nal}$ crystal a $\mathrm{Ge}(\mathrm{Li})$ detector observes the catcher foil. During flight all but the isomeric states de-excite to the ground state. Should the isomeric state subsequently decay by a cascade, in the most favourable case, all the $\gamma$ quanta are detected in either the $\mathrm{Ge}(\mathrm{Li})$ or the Nal where a summed peak is received. By triggering the $\mathrm{Ge}(\mathrm{Li})$ with suitable $\mathrm{Nal}$ events, all the members of the cascade and the energy of the isomeric state are yielded. The latter can be taken directly from the $\mathrm{Nal}$, for if the $\mathrm{Ge}(\mathrm{Li})$ spectrum is used, troubles are encountered from the complicated spectrum of competitive transitions. By timing the event against a pulsed beam, the half life of the isomer is also given. By these experiments, the expected high spin isomers have been found in the Gd-region.

Andreas Bockisch

\section{Quarks and Jets}

(An abridged version of the original manuscript)

The best way (if not the only way) to understand strong interaction dynamics is by the scattering of particles at high energies. The hope is that in so doing one actually probes the hadronic structure and interacts with the hadronic constituents (i.e. quarks) directly. To illustrate this, we mention the following two very important sets of experiments:-

(i) deep inelestic scattering of leptons off hadrons, and

(ii) "quark jets" and "gluon jets".

(i) In experiments done in 1968/9 at SLAC, quarks/partons were actually "seen" inside the nucleons for the first time. Briefly, by scattering very high-energy electrons off nucleons, the nucleon was shown to consist of two ingredients: an electrically neutral "glue" ( $~ 50 \%$ ) and three point like objects which most probably carry exactly the quark quantum numbers. These are called the "valence quarks". It is also believed that there is a "sea" or "soup" of qव pairs inside the nucleon(s). These observations and late experiments with deep inelastic scattering of neutrinos off nuc- leons (through weak interactions) demonstrated the following two highly significant points:-

(a) at presently available energies, these constituents behave as if they are point-like objects which leads us to the concept of scaling;

(b) at very high energies and large momentum transfers the force between quarks, when they are "very close" to each other is almost zero and the closer they get to each other the freer they become-hence the term: "Asymptotic Freedom". (N.B. Q.CD requires this too).

(ii) The closest one has got so far in trying to observe free quarks is in the production of jets. The first definite indication of "jet structure" was found in 1975 (and confirmed later) in $\mathrm{e}+\mathrm{e}-$ annihilation at the SPEAR storage rings at centre of mass energies above the $\mathrm{J} / \Psi$ resonance region (see Fig. 1).

To search for jets, they first calculated the tensor $\mathrm{Tij}$ for each event: $T^{i j}=\sum_{n}\left(S^{i j} \vec{p}_{n}^{2}-p_{n}^{i} p_{n}^{j}\right)$. i and $j$ refer

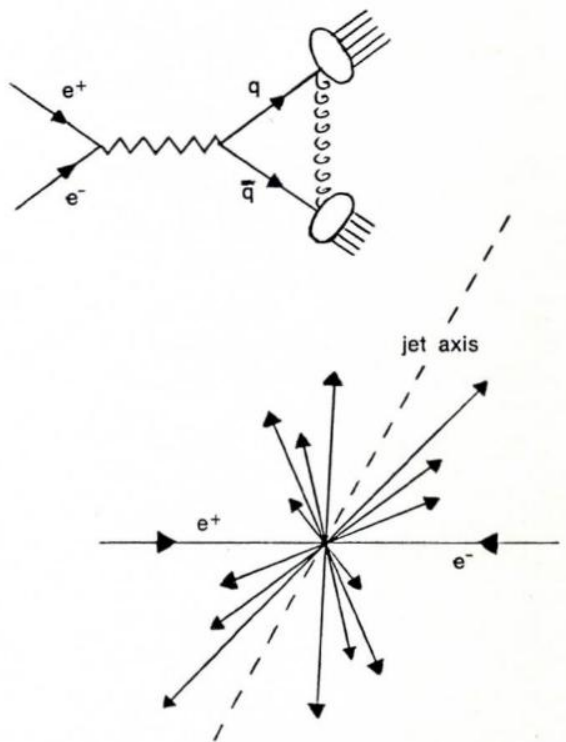

Fig. 1 (Above) Electron-positron annihilation into two quark jets showing the final state interaction;

(below) Example of jets in $e^{+-e^{-}}$annihilation.

to the spatial components of each particle with momentum $\vec{p}_{n}$ and the summation is over all the detected particles $(n=1,2, \ldots)$ in the event. Diagonalizing $\mathrm{T}^{\mathrm{ij}}$ we get the principal moment eigenvalues $\lambda_{1} \lambda_{2}$ and $\lambda_{3}$ in momentum space. Subsequently one defines the sphericity $S$, by $S=$ 